\title{
Las WebQuest, una Propuesta de Formación Docente para Propiciar el Desarrollo de Competencias en los Alumnos de Ingeniería
}

\author{
Silverio Pérez-Cáceres*, Alfredo Cristóbal-Salas, Raúl Varguez-Fernández y \\ Efrén Morales-Mendoza \\ Fac. de Ingeniería en Electrónica y Comunicaciones, Universidad Veracruzana, \\ Prolongación de Avenida Venustiano Carranza s/n, Colonia Revolución, Poza Rica, \\ Veracruz-México (email: sperez@uv.mx; acristobal@uv.mx; rvarguez@uv.mx; efmorales@uv.mx) \\ * Autor a quien debe ser dirigida la correspondencia.
}

Recibido Ene. 15, 2011; Aceptado Feb. 25, 2011; Versión final recibida Mar. 14, 2011

\section{Resumen}

Se presenta y discute el uso de las WebQuest (Búsqueda en la Web), como estrategia innovadora en el proceso de enseñanza-aprendizaje de estudiantes universitarios. Se sostiene que existe la necesidad de formación del personal docente en estrategias basadas en Tecnologías de Información y Comunicación (TIC), que propicien el desarrollo de competencias en alumnos de Ingeniería. Se realizó un taller sobre la teoría de las WebQuest impartido a docentes de la Universidad Veracruzana en México, quienes diseñaron y elaboraron WebQuest relacionadas con una asignatura que imparten. Los docentes aplicaron criterios de calidad para la elaboración de WebQuest y evaluaron y discutieron el funcionamiento de las mismas en una sesión grupal. El grupo de discusión concluyó que el uso de las WebQuest en el desarrollo de competencias es viable.

Palabras clave: WebQuest, desarrollo de competencias, TIC, formación docente, enseñanzaaprendizaje

\section{WebQuest, a Proposal for Training Instructors to support the Development of Competences in Engineering Students}

\begin{abstract}
The use of WebQuest, an innovative strategy in the teaching-learning process of undergraduate students, is presented and discussed. This paper also states that there is a need for teacher training in strategies based on Information and Communications Technologies (ICT) that promote skill development in engineering undergraduate students. A workshop about the theory of WebQuest was offered to teachers of the School of Engineering in Electronics and Communications of the Universidad Veracruzana in Mexico who designed and developed WebQuuest related to a course that they teach. The professors applied quality criteria for the development of the WebQuest and discussed and evaluated their functioning in a group session. The group discussion concluded that the use of WebQuest is feasible in developing competences.
\end{abstract}

Keywords: WebQuest, development of competences, ICT, teacher training, teaching-learning 


\section{INTRODUCCIÓN}

En la actualidad existen distintas iniciativas, en diversos ámbitos, relativas a la educación basada en competencias así como al uso de las Tecnologías de Información y Comunicación (TIC) en la educación: a nivel internacional son conocidos los trabajos realizados en el marco de los proyectos Tuning y 6x4 UEALC y la definición de los estándares de competencias en TIC para docentes publicados por la UNESCO; En México, los programas de educación básica, media y media superior han iniciado planes de estudios basados en competencias, en este ámbito la Secretaría de Educación Pública (SEP) y la Asociación Nacional de Universidades e Instituciones de Educación Superior (ANUIES) implementaron un Diplomado denominado "Competencias docentes en el nivel medio superior", con el objetivo de que los profesores de educación media superior integren a su práctica docente estrategias innovadoras basadas en la construcción de competencias. Además se han implementado, en diversas instituciones de nivel superior, modelos basados en competencias propiciando el uso de las TIC, este es el caso de la Universidad Veracruzana (UV), que desde 1999 inició un modelo educativo centrado en el aprendizaje denominado actualmente Modelo Educativo Integral y Flexible (MEIF) que considera, además el enfoque basado en competencias, el uso de la tecnología en el aula. Con la intención de asegurar el cumplimiento de estos principios, inicia en 2009 el Proyecto Aula, que tiene como objetivo la formación de comunidades y redes de académicos orientadas a la innovación de las prácticas docentes en el aula, centradas en el aprendizaje de los estudiantes y en la construcción colectiva del conocimiento.

En la entidad en la que se realiza este proyecto de investigación se imparte la carrera de Ingeniería en Electrónica y Comunicaciones, con un Plan de Estudios basado en competencias desde Agosto del 2004 acorde a los lineamientos del MEIF de la UV, con el cual se pretende desarrollar ocho competencias: Aplicación, Implementación, Control, Planeación, Investigación, Diseño, Análisis y Evaluación. Con la finalidad de indagar sobre el cumplimiento de los objetivos planteados en el Plan de Estudios, se realizó en el 2009 un diagnóstico de la implementación del Plan de Estudios a partir de observaciones de clase y una encuesta al personal docente: en las observaciones de clase no se detectó el uso de metodologías basadas en competencias y un escaso uso de estrategias basadas en TIC; la encuesta aplicada a 15 de 40 profesores arrojó los siguientes resultados: el $86.67 \%$ respondió que sus programas están basados en competencias, el $73.33 \%$ respondió que aplica estrategias basadas en competencias, el $60 \%$ aplica instrumentos de evaluación basados en competencias; el $80 \%$ respondió que utiliza materiales audiovisuales, el $60 \%$ utiliza software de simulación, sólo el $13.33 \%$ reportó el uso de manejadores de contenido para cursos en línea y el $20 \%$ utiliza estrategias basadas en TIC. Considerando lo anterior se realizó la tarea de investigar la aplicación de estrategias basadas en TIC que propicien el desarrollo de competencias y se seleccionó a las WebQuest.

El modelo de Webquest (Dodge, 1995) fue definido como una actividad orientada a la investigación en la cual toda o casi toda la información con la que los alumnos interactúan proviene de recursos en la Internet. Utiliza una tarea auténtica para motivar la investigación por parte del alumnado de una pregunta central, con un final abierto, el desarrollo de su conocimiento individual, y la participación en un proceso final de grupo, con la intención de transformar la información recién adquirida en un conocimiento más sofisticado. Las mejores WebQuest hacen esto de una forma que motiva al alumnado a ver relaciones temáticas más enriquecedoras, facilitan la contribución al mundo real del aprendizaje y reflexiona sobre sus propios procesos metacognitivos (March, 2003).

Las WebQuest son generalmente construidas alrededor de un escenario de interés para los estudiantes quienes trabajan en pequeños grupos siguiendo los pasos definidos en ella para: examinar los problemas, proponer hipótesis, buscar información, analizar y sintetizar la información con la ayuda de preguntas orientadoras y finalmente para presentar soluciones a problemas (Zheng, 2008). Una WebQuest, se compone de seis partes esenciales: Introducción, Tarea, Proceso, Recursos, Evaluación y Conclusión. Las WebQuest guían al estudiante a completar una tarea o resolver un problema que lo conduce a un pensamiento de alto orden y no sólo a la simple búsqueda y recuperación de información (Gülbahar et al., 2010); Vidoni y Maddux 
(2002) apoyan también este pensamiento al asegurar que las WebQuest "retan a los estudiantes" de manera intelectual y académica en lugar de sólo desarrollar en ellos una competencia de búsqueda de información en la Web. Algunos artículos mencionan la importancia de las WebQuest, como Cegarra (2008), que realiza una revisión teórica y metodológica de la WebQuest como estrategia constructivista de aprendizaje basada en Internet.

March (1998) agrupó a las WebQuest bajo tres rubros: la motivación del estudiante y su autenticidad, el desarrollo de la competencia de pensamiento y el aprendizaje cooperativo. Posteriormente Zheng et. al. (2005), consideraron que las WebQuest descansan sobre cuatro pilares: aprendizaje con andamiaje, pensamiento crítico, competencias sociales y aplicación del conocimiento. Por otro lado, Leahy y Twomey (2005) discuten sobre dos pilares adicionales de las WebQuest: el fortalecimiento de la motivación y la construcción de actividades colaborativas. En Jonassen et al. (2003) se señala que las WebQuest son un buen punto de inicio para los profesores que comienzan a utilizar el Internet como herramienta didáctica debido a que les provee de una estructura claramente definida en el que su diseño y uso están fundamentados.

Por otro lado, March (2004) asegura que las WebQuest usan el Internet de manera efectiva para promover las prácticas basadas en procedimientos. Adicionalmente, Cramer (2007) comenta que las WebQuest son una forma efectiva para enganchar a los estudiantes en una instrucción auténtica en la cual las actividades orientadas a la búsqueda de conocimiento usan la Web para la resolución de una problemática. Existen trabajos en todos los niveles educativos, en Preescolar podemos mencionar a Aguiar y Cuesta (2009) quienes concluyen que "las Webquests facilitan el desarrollo de múltiples habilidades perceptivo-cognitivas gracias al manejo de Internet" y "además de suponer un potente instrumento por el que introducir las TIC en el aula, permite el desarrollo de actividades con las que desarrollar múltiples habilidades en diferentes áreas".

\section{LA WEBQUEST COMO HERRAMIENTA DIDÁCTICA}

En años recientes, la tecnología ha ido incrementando su contribución a la educación desde dos perspectivas: como herramienta para el incremento de la efectividad del proceso enseñanzaaprendizaje y para fortalecimiento de las competencias del estudiante en el uso de la tecnología. Hoy en día, la investigación sobre nuevas formas de contribución y el impacto de la tecnología en la educación siguen como temas activos. En este sentido, las WebQuest se han posicionado como un nicho de investigación debido a que agrupa el aprendizaje basado en tareas en un ambiente digital (Stoks, 2010).

Gülbahar et al. (2008), muestra a las WebQuest como una propuesta sistemática y orientada al estudiante para la enseñanza de un tema específico en un ambiente mayoritariamente dominado por la búsqueda de información en la Web a través de estrategias de aprendizaje activo y colaborativo donde se pueden compartir experiencias y nuevo aprendizaje alcanzado por alguno de los miembros del equipo de trabajo. En (Zheng et al., 2008), se presenta a las WebQuest como un modelo instruccional que ha sido adoptado por su facilidad de diseño, desarrollo y uso en los salones de clase. En un estudio realizado por Milson y Downey (2001) concluyeron que las WebQuest ayudan a los estudiantes a tener un aprendizaje significativo y dinámico al trabajar con un equipo que puede resolver problemas del mundo real, facilitan el aprendizaje efectivo al proveer de recursos estructurados de tal manera que los aprendices no tienen que "gastar tiempo en búsquedas infructuosas" y por último, permiten a los maestros usar de manera efectiva los recursos computacionales dentro del salón de clase. Por otro lado, Perkins y McNight (2005) explican que uno de los beneficios de las WebQuest sucede durante el proceso de resolver un problema, donde los estudiantes aprenden competencias académicas de una forma interactiva al involucrarse y no de manera aislada. Además Schweizer y Kossow (2007) observaron que con las WebQuest los estudiantes se motivaban a aprender nuevo conocimiento enganchándose en un pensamiento de alto orden que adquirían al completar la tarea y encontraron que los estudiantes apreciaban el aprendizaje colaborativo en donde contribuían de manera colectiva para el éxito del proyecto. 
En Gülbahar et al. (2008) se reporta que los resultados de los aspirantes a maestros, quienes diseñaron WebQuest como parte de un curso experimental, "fueron positivos en el uso" de las WebQuest en su profesión, mencionando que las WebQuest motivan la participación de los estudiantes en los proyectos, les ayudan a trabajar de una manera planeada/guiada y los motivan a completar las tareas a tiempo apoyándoles con conocimiento teórico en diferentes situaciones. En otro estudio, el desarrollo de las WebQuest, desde la perspectiva del docente, mejora la productividad de los estudiantes y mejora su entendimiento sobre la manera de integrar la tecnología en el salón de clases de una manera constructivista (Kundu y Bain, 2006). Como se sostiene en el artículo (Lamb, 2004), más que escribir un ensayo o hacer una presentación para el maestro, las WebQuest requieren que los estudiantes conecten su entendimiento de la información en situaciones significativas a través de productos para audiencias reales.

Reyes y Gálvez (2010), en una investigación de experiencias docentes de la asignatura de Materiales de Construcción, concluyen que "nuevos métodos activos de enseñanza utilizados (aprendizaje cooperativo de problemas, trabajo en grupo, uso de nuevas tecnologías como la plataforma Moodle, evaluación continua y tutorías concertadas) ayudan al aprendizaje, y a las oportunidades que los alumnos tienen de aprender motivados"; Del Moral y Villalustre (2007), en una experiencia de teleinformación sustentada en el trabajo autónomo de los estudiantes, de la asignatura de "Educación Virtual en el ámbito rural (Rur@Inet)", a través de la adopción de un nuevo modelo de enseñanza centrado en el desarrollo de novedosas estrategias didácticometodológicas basadas en el trabajo colaborativo de investigación a través de Webquest, concluyen que "se ha llevado a cabo un proceso de transformación que afecta tanto a la presentación de contenidos y a la integración de actividades que favorecen el trabajo colaborativo, como a las propuestas de evaluación continua", esto en atención a "las nuevas necesidades formativas en el ámbito universitario, enunciados en Bolonia: un mayor énfasis en el aprendizaje autónomo de los estudiantes, la apuesta por un enseñanza centrada en el discente y el diseño de materiales flexibles que faciliten el aprendizaje autónomo".

Rodríguez y Escofet (2006), plantean el desarrollo de contenidos digitales por los estudiantes de distintas asignaturas del nivel universitario, en tres tipos: libros electrónicos, relatos digitales y WebQuest, está última como actividad didáctica que plantea una navegación guiada por Internet para conseguir un objetivo educativo concreto mediante la indagación. En sus conclusiones comentan que "El hecho de permitir que los estudiantes se coloquen un una posición de productores de conocimientos, demuestren que sus competencias adquiridas y trabajen, en definitiva, en un proyecto integrado (en el que las tecnologías no están alejadas formalmente de los contenidos) es un concepción pedagógica que creemos interesante por el cambio de metodología que supone y relativamente poco habitual en estudios de Ciencias Sociales y Humanidades". En una investigación realizada en el marco de la experiencia de innovación docente con TICs llevada a cabo en la Universidad de Jaume I en España, para la adaptación de la docencia al Espacio Europeo de Educación Superior (EEES) mediante WebQuests (Bernabé, 2009), se concluye que "la utilización de la metodología WebQuest es adecuada para llevar a cabo la docencia centrada en el alumnado y enfocada al desarrollo de competencias característica del EEES. De manera que el profesorado universitario tiene en las WebQuests un recurso muy adecuado para incorporar algunas de las principales competencias más valoradas en el EEES".

En la Facultad de Educación de la Universidad de Valladolid (Santos et al., 2010) organizaron la asignatura de Nuevas Tecnologías Aplicadas a la Educación, apoyada en una plataforma Wiki, proponiendo al alumnado una serie de actividades que debían realizar colaborativamente, como las Wiki-WebQuest; los autores señalan que algunos de los aspectos de la experiencia realizada que han tenido implicaciones educativas relevantes para el alumnado de Magisterio, como en "la aplicación del aprendizaje a contextos profesionales reales, la transferencia de los conocimientos de la universidad a los colegios, el compromiso y responsabilidad social del alumnado de la universidad al trabajar con alumnado de primaria y, de este modo, sensibilizarse con el ámbito educativo y la formación crítica del alumnado universitario en relación con las TIC" son: "vivir la experiencia en primera persona, comprobar lo que funciona y lo que no y, sobre todo, aprender el uso educativo de las TIC, es decir, su verdadera utilidad en el aula". 
En otro trabajo se muestran algunos usos de la red en la enseñanza de la química general y química analítica, usando WebQuest para los cursos de postgrado de Metodología de la Investigación Científica e Investigación Educativa (Torres et al., 2008). En una investigación realizada con 63 universitarios y profesionales de logopedia, psicología y pedagogía que, utilizando herramientas informáticas, han de resolver once casos reales de niños y adultos no vocales que buscan introducir mejoras en sus sistemas de comunicación aumentativa (Arriba, 2008), en el cual se comparan dos contextos de aprendizaje en la consecución del conocimiento experto elaborados con un formato de WebQuest; los autores discuten la necesidad de facilitar herramientas informáticas de comunicación que permitan a alumnos y profesionales expertos crear espacios de trabajo compartido para abordar casos reales de especial complejidad.

En cuanto al diseño e implantación de cursos dirigidos a docentes de educación superior (Fracchia et al., 2010), presentan su experiencia con el curso "TICs en educación" con el objetivo de que los docentes puedan incorporar las nuevas tecnologías de información y comunicación (TICs) en el aula, diseñando actividades para que se aprenda sobre las TICs usando como medio de enseñanza las mismas TICs. De sus resultados comentan que "los alumnos al trabajar con las TICs pudieron vivenciar aspectos que se desprenden de su rol de alumno, del trabajo realizado por los tutores y que le ayudarán a comprender mejor su futuro rol docente, además de las implicancias que generan los avances de la Tecnología". Comentan además que los diferentes tipos de herramientas tecnológicas utilizadas (como WebQuest), el entorno tecnológico provisto y sus aplicaciones, facilitarán a los alumnos la construcción de propuestas de proyectos interdisciplinarios, en los que tendrán que seleccionar y usar adecuadamente las TICs en función de las necesidades detectadas o problemas presentados, confirman además la necesidad de construir equipos de trabajo comprometidos con la modalidad, que aporten las demandas espontáneas de los cursantes y con una importante formación no sólo técnica sino también pedagógica y didáctica.

Finalmente, se dispone en la Internet de varias herramientas para la elaboración o generación de WebQuest como 123 tu webquest, Proyecto Hormiga, Edutic (Universidad de Alicante), Generador de la Comunidad de Aragón, Webquest Generator, GardenQuest y Teacher Webquest, las cuales fueron analizadas por Temprano y Gallego (2009) para diseñar e implementar un nuevo generador de WebQuest, al cual denominaron "PHP Webquest", con la intención poner a disposición del colectivo docente una herramienta más dentro del ámbito del Software Libre para facilitar la práctica educativa, en este artículo mencionan que se han registrado 3,400 usuarios en el sitio Web de 700 Instituciones Educativas creando más de 4,500 actividades en phpwebquest.org.

Por otro lado, se han encontrado algunas desventajas de las WebQuest, en (Stoks, 2010) se menciona que las WebQuest pueden llegar a ser consumidoras de tiempo tanto en su creación como en su ejecución, además éstas deben ser siempre adaptadas al contexto del estudiante para asegurarse que sean comprensibles; otro punto criticado de las WebQuest es que los enlaces en Internet se desactualizan rápidamente y los enlaces suelen extinguirse en el corto plazo; en (Westhoff, 2004) los maestros se mostraron indecisos en los efectos de las WebQuest sobre todo en el momento de evaluar los trabajos argumentando que en las WebQuest no todos los estudiantes adquieren el mismo conocimiento sino sólo el conocimiento asociado al problema que resuelven, como también se opina en (March, 1998). Además, los docentes piensan que han invertido mucho tiempo que pudieron haber invertido en la preparación de las clases; incluso, algunos docentes argumentaron que las WebQuest distraen a los estudiantes del análisis de la literatura oficial del curso. En Zeng et al. (2008) se discute que el diseño y desarrollo de las WebQuest es un proceso complejo que involucra una planeación cuidadosa al poner en perspectiva todas las variables que influyen en la capacidad de aprender del estudiante la cual debe incluir el factor social, psicológico, cognitivo y de desarrollo entre otras. En Gülbahar et al. (2008) se discute un aspecto crucial negativo respecto de las WebQuest, la disponibilidad y efectividad de las condiciones técnicas del Internet para facilitar la búsqueda de información, aseguran que teniendo una red de interconexión poco optimizada o limitada puede resultar desmotivante para los estudiantes. 
Si bien, como se muestra en las estadísticas anteriores, se han creado un gran número de WebQuest y se han realizado un número considerable de investigaciones del impacto de las mismas en el proceso de enseñanza-aprendizaje, las cuales disminuyen cuando se trata de su aplicación en el nivel de Enseñanza Superior y sobre todo en la enseñanza de la Ingeniería, la aplicación de las WebQuest en la Educación Superior sigue siendo fuente inspiradora de investigación al tratar de introducir, de manera controlada, al Internet como herramienta didáctica, requiriéndose de mayor experimentación y exploración. Esta estrategia representa una oportunidad de formación e innovación docente, además de posibilitar la investigación acerca de la percepción de esta estrategia tanto de los profesores como de los alumnos.

Derivado de lo anterior se plantea la siguiente pregunta de investigación: ¿Cuál es la apreciación del personal docente sobre el uso de las WebQuest en el desarrollo de competencias en los alumnos de Ingeniería?. Para guiar el proceso de investigación se plantea el siguiente objetivo: "Implementar un programa de formación docente para experimentar en el diseño de WebQuest, que conlleve a valorar la posibilidad de desarrollo de competencias al ser utilizadas en el proceso de enseñanza-aprendizaje de estudiantes universitarios". El método utilizado fue la investigación acción, el cual se describirá más a detalle en la siguiente sección.

\section{METODOLOGÍA}

Para responder a la pregunta de investigación, primeramente se diseñó e impartió un taller de capacitación al personal docente. Al final se integró un grupo de discusión, como técnica para la recolección de datos, indagando sobre la apreciación del personal docente acerca del desarrollo de competencias utilizando WebQuest. Este proceso corresponde a la primera etapa de un proyecto de investigación en el que se ha evaluado el desarrollo de competencias en los alumnos de Ingeniería utilizando WebQuest, con resultados muy alentadores que serán presentados en otro momento; El proyecto completo se ha concebido en el paradigma cualitativo, considerando sus características más relevantes, expresadas por Hernández et al. (2006): i) se utiliza primero para definir y refinar preguntas de investigación (Grinnell, 1997); ii) en la mayoría de los estudios no se prueban hipótesis; iii) la recolección de los datos con instrumentos no estandarizados consiste en obtener las perspectivas y puntos de vista de los participantes; iv) dentro de las técnicas para la recolección de datos se encuentra la discusión en grupo. El método de investigación se basa en la investigación-acción, dado que la problemática se resolverá con la participación de los alumnos y profesores en su entorno natural que es el aula y una de las características principales es la transformación y mejora de la realidad educativa. En particular, se seleccionó el diseño práctico por las características de Creswell (2005), señaladas en Hernández et al. (2006).

El taller se tituló "Las WebQuest en el desarrollo de competencias" registrado y validado por dos dependencias institucionales, el Departamento de Educación Continua de la Dirección General de Tecnologías de Información y la Dirección General del Área Académica Técnica. Asistieron 8 docentes de la Facultad de Ingeniería y el taller tuvo una duración de 30 horas presenciales, más las dedicadas por los asistentes a lecturas y diseño de materiales; se utilizó el manejador de contenidos "Claroline". El taller se llevó a cabo de forma participativa exponiendo temas introductorios los mismos participantes, los docentes asistentes diseñaron sus propias WebQuest y los temas seleccionados fueron de las asignaturas de ecuaciones diferenciales, investigación de operaciones, sistemas operativos, programación orientada a objetos, procesamiento analógico de señales, microprocesadores y microcontroladores; en el diseño de las WebQuest se consideraron los criterios de calidad. Las WebQuest fueron presentadas en plenaria por cada uno de los asistentes, evaluando de forma grupal el funcionamiento de las mismas. La evaluación del taller se realizó utilizando el formato de encuesta definido institucionalmente.

Al final, para indagar sobre la apreciación de los asistentes acerca del uso de las WebQuest en el desarrollo de competencias, se integró la sesión de grupo de discusión basado en Krueger (1991), con el propósito de realizar la evaluación de una nueva idea para un programa de intervención. La sesión tuvo una duración de dos horas basándose en la guía diseñada para su conducción. La 
sesión fue grabada en audio y video, con la autorización de los asistentes, para el posterior análisis de la discusión.

Algunas características de la innovación educativa (Pascual, 1998), consideradas en la propuesta de formación, son: un proceso participativo, se tiene la mirada puesta en llegar a lo ideal con la aplicación de estrategias, potenciar el uso de las TIC tanto en el taller como en la posible aplicación de las WebQuest con los alumnos, no se queda en buenas intenciones, porque se lleva a la práctica desde el mismo taller diseñando la estrategia e induciendo al personal docente en su aplicación con los alumnos, finalmente se espera articular en el proceso a todos los implicados, desde el personal directivo, los docentes y sobre todo una concientización a los alumnos del nuevo rol y las nuevas exigencias.

\section{RESULTADOS Y DISCUSIÓN}

Se presentan los resultados de la encuesta como parte del proceso de evaluación del taller y del grupo de discusión como parte de la investigación. Las secciones de la encuesta institucional de evaluación del taller de mayor interés para esta investigación son la del desempeño del instructor (capacidad de manejo del grupo, conocimientos del tema, uso de apoyos didácticos, habilidad didáctica pedagógica, manejo del tiempo) y la del contenido del curso (originalidad de conceptos, congruencia con los objetivos planeados, calidad general del material expuesto). Los docentes evaluaron tanto el desempeño del instructor como el contenido del curso de excelente a bueno, no presentándose valoraciones de regular y malo.

A continuación se presentan los resultados del grupo de discusión, sesión realizada el 28 de enero de 2010, en formato descriptivo. La discusión se inició explorando como concebían los docentes las WebQuest y que posibilidades habría de utilizarlas en el proceso enseñanza-aprendizaje. Los comentarios más relevantes se presentan en lo que sigue, identificando a los participantes por la primera letra de su nombre. Se consultó también la opinión con respecto al desarrollo de competencias con WebQuest, el fomento de valores y la evaluación basada en competencias.

\section{S: "Es un estrategia didáctica interesante"}

$R$ : "Alternativa para aprovechar la red más eficientemente".

$L$. "Es un método de enseñanza y herramienta de aprendizaje"

$T$. "Innovador, interesante y abrumador, ¿de verdad nos servirá?"

$A$. "No le veo nada interesante, es una guía de prácticas, de laboratorio, un cheklist, no hay nada de innovación detrás de esto".

$R$. "Tal vez no sea innovador, pero habría que establecer el posible impacto; acercarlas a la enseñanza basada en proyectos". "Si se planea de manera colegiada puede tener un impacto mucho más eficiente".

L. "Lo único innovador sería lo que hacemos nosotros";

A. "Sería un parte aguas, si eliminamos todos lo cursos presenciales y lo hacemos todo con WebQuest, están pensadas para que el maestro pase a ser un asesor y no instructor".

C. "Sin necesidad del maestro tú puedes hacer lo que está indicado, a los alumnos les interesa aprender con ejemplos cotidianos".

A. "siendo realistas, a lo mejor si nos ayudan, en el sentido de que no necesito explicar el procedimiento".

En lo que respecta al fomento de las TIC utilizando las WebQuest, las opiniones fueron las siguientes:

A. "No es necesario, puedes hacerlo en papel, entregar copias, no te obligan las WQ a usar las TIC".

$R$. "El concepto de WQ no implica que vas a usar eficientemente las TIC, depende mucho de cómo la plantees, habría que ver el factor de calidad, los estándares de calidad". 
L. "Haciendo la primera presentación, las demás ya te salen".

$T$. "Uno tiene la idea, pero le cuesta mucho trabajo plasmarla"

S. "Depende del que lo va aplicar, si requiere aplicar las TIC".

La opinión al respecto del desarrollo de competencias con WebQuest, el fomento de valores y la evaluación basada en competencias:

A. "No".

$R$. "Fomenta competencias tal vez, garantiza no creo. Fomenta las competencias si pones las reglas".

S. "Valores si pones tiempo de entrega a las actividades"

$T$. "Fomenta algunos valores como responsabilidad"

$L$. "El proceso de evaluación por competencias es muy complicado"

$R$. "Las reglas de las WQ indican que vas a realizar una evaluación real"

Finalmente, todos los docentes manifestaron su intención de aplicar las WebQuest en el siguiente período semestral. Como se observa, a las WebQuest se les percibe de distintas formas, como metodología, estrategia, herramienta o un simple listado; y en su mayoría las consideran como innovadoras, con algunas opiniones que las consideran como una simple guía. En lo que respecta a las posibilidades de uso, los docentes en su mayoría consideran que es viable su uso en el proceso de enseñanza-aprendizaje. En lo que respecta al uso de las TIC, existe cierta reserva, considerando importante la planeación de los cursos. Finalmente, en lo que respecta al desarrollo de competencias (incluyendo los valores como parte de las actitudes, tercer elemento del concepto de competencia), existen opiniones encontradas: quienes opinan que no propician el desarrollo de competencias, mientras que otros consideran que es posible si se realiza una planeación adecuada, estableciendo reglas claras.

\section{CONCLUSIONES}

La WebQuest representa una oportunidad para la innovación educativa, como parte de la formación docente así como en el uso de las WebQuest en el desarrollo de competencias de los alumnos de Ingeniería.

Adicionalmente, el proceso llevado a cabo para definir el programa de formación docente y la evaluación de la estrategia WebQuest, representa un aporte metodológico que puede ser replicado para otras temáticas de formación y evaluación de herramientas basadas en TIC.

La experiencia de implementación del taller propició tanto en el instructor como en los docentes participantes, el uso de las TIC, como correo electrónico, navegadores, presentaciones con diapositivas y manejadores de contenido para cursos en línea.

La apreciación positiva del personal docente ha inducido al desarrollo y aplicación de las WebQuest por cuatro docentes, lo que representa la oportunidad de continuar con la investigación, evaluando el impacto en el desarrollo de competencias en los alumnos de Ingeniería.

\section{REFERENCIAS}

Aguiar, M.V. y Cuesta, H. Importancia de trabajar las TIC en educación infantil a través de métodos como la Webquest. Pixel-Bit. Revista de Medios y Educación, (34), 81-94 (2009).

Arriba, J.A. Aprendiendo a resolver casos reales mediante la utilización de herramientas informáticas de aprendizaje y colaboración. RU\&SC. Revista de Universidad y Sociedad del Conocimiento, 5(2), 36-48 (2008). 
Bernabé, I. Recursos TICs en el Espacio Europeo de Educación Superior (EEES): las Webquests. Pixel-Bit. Revista de Medios y Educación, (35), 115-126 (2009).

Cegarra, J. WebQuest: Estrategia constructivista de aprendizaje basada en Internet. Investigación y Postgrado, 23(001), 73-91 (2008).

Cramer, S.R. Update Your Classroom with Learning Objects and Twenty-First-Century Skills. The Clearing House, 80 (3), 126-126 (2007).

Creswell, J. Educational research: Planning, conducting, and evaluating quantitative and qualitative Research, $2^{\mathrm{a}}$ edición, 552-552, Pearson Ed. Inc., Upper Saddle River, USA (2005)

Del Moral, M.E. y Villalustre, L. Ruralnet: Prácticas virtuales de aprendizaje colaborativo a través de WebQuest. Pixel-Bit.Revista de Medios y Educación, (029), 25-35 (2007).

Dodge, B. WebQuests: a technique for Internet-based learning. Distance Educator, 1(2), 10-13 (1995).

Fracchia, C.C., Alonso de Armiño, A. y Plaza, M.J. Formación de Docentes: uso de TICS en los procesos de enseñanza, Segundas Jornadas de Educación Mediada por Tecnología "El cambio educativo", 205-212, Buenos Aires, Argentina, 12 y 13 de marzo (2010).

Grinnell, R.M. Social word research \& evaluation: Quantitative and qualitative approaches. $5^{a}$ edición, E. E. Peacock Publishers, Ithaca, USA (1997).

Gülbahar, Y., Kalelioğlu, F., y Madran, R. O. Using dynamic WebQuest approach in teacher education to promote project-based learning, Proceedings of 8th International Educational Technology Conference, 1156-1159, Eskişehir, Turkey, 6 al 9 de Mayo (2008).

Gülbahar, Y., Madran, R. O., y Kalelioglu, F. Development and Evaluation of an Interactive WebQuest Environment: "Web Macerasi". Educational Technology \& Society, 13 (3), 139-150 (2010).

Hernández, R., Fernández, C. y Baptista, P. Metodología de la Investigación, 4a edición, 8-9, McGraw-Hill, México (2006).

Jonassen, D.H., Howland, J., Moore, J., y Marra, R.M. Learning to Solve Problems with Technology: A Constructivist Perspective. USA: Pearson Education, Inc. (2003).

Krueger, R.A. El Grupo de discusión. Guía práctica para la investigación aplicada. Pirámide, Madrid, España (1991).

Kundu, R. y Bain, C. WebQuests: Utilizing Technology in a Constructivist Manner to Facilitate Meaningful Preservice Learning. Art Education, 59(2), 6-11(2006).

Lamb, A. WebQuests. School Library Media Activities Monthly. 21(2), 38-40 (2004).

Leahy, M. y Twomey, D. Using web design with pre-service teachers as a means of creating a collaborative learning environment. Educational Media International, 42(2), 143-151 (2005).

March, T. Why WebQuests? An introduction, (1998), http://tommarch.com/writings/intro_wq.php. Acceso: 7 de marzo (2011).

March, T. The learning power of WebQuest. Educational Leadership, 61(4), 42-47. (2003).

March, T. The Learning Power of WebQuests, (2004). http://tommarch.com/writings/wq_power.php Acceso: 7 de marzo (2011) 
Milson A.J. y Downey P. WebQuest: using Internet resources for cooperative learning. Social Education (65), 144-146 (2001).

Pascual, R., La gestión educativa ante la innovación y el cambio, Narcea, Madrid, España (1998).

Perkins, R., y McKnight, M. L. Teachers' attitudes toward WebQuests as a method of teaching. Computers in the Schools, 22(1), 123-133 (2005).

Reyes, E. y Gálvez, J.C. Experiencias Docentes en Innovación Educativa como Mejora de una Enseñanza Tradicional de los Materiales de Construcción, Formación Universitaria, 3(4), 13-24 (2010).

Rodríguez, J.L. y Escofet, A. Aproximación centrada en el estudiante como productor de contenidos digitales en cursos híbridos. Univ. y Sociedad del Conocimiento. 3(002), 20-28 (2006).

Santos, R. y otros tres autores. La wiki-webquest: una actividad colaborativa en la asignatura de Nuevas Tecnologías Aplicadas a la Educación. Rev.de Educación a Distancia, X(XII), 1-15 (2010).

Schweizer H. y Kossow B. WebQuests: tools for differentiation. Gifted Child Today 30, 29-35 (2007).

Stoks, Gé. WebQuests in the Training of Teachers of Modern Languages. CORELL: Computer Resources for Language Learning 3, 25-28. (2010).

Temprano, A. y Gallego, D.J. Diseño, desarrollo e implantación de un software libre para la creación de Webquest. Pixel-Bit. Revista de Medios y Educación, (34), 165-177 (2009).

Torres-Pérez, D., Castro-Calleja, M.T. y Llitjós-Viza, A. Ejemplos del uso de Internet en la enseñanza de la Química. Pedagogía Universitaria, 13(2), 35-46 (2008).

Vidoni, K. L., y Maddux, C. D. WebQuests: Can they be used to improve critical-thinking skills in students?. Computers in the Schools, 19(1/2), 101-117 (2002).

Westhoff, W. The art of playing a pinball machine. Babylonia 3(04). 58-62 (2004).

Zheng, R., Stucky, B., McAlck, M., Menchana, M., y Stoddart, S. WebQuest learning as perceived by higher-education learners. TechTrends, 49(4), 41-49 (2005).

Zheng, R., Pérez, J., Williamson, J., y Flygare, J. WebQuests as perceived by teachers: implications for online teaching and learning. Journal of Computer Assisted Learning, (24), 295304 (2008). 